\title{
Slipping Objects in Image Registration: Improved Motion Field Estimation with Direction-Dependent Regularization
}

\author{
Alexander Schmidt-Richberg, Jan Ehrhardt, Rene Werner, and Heinz Handels \\ Department of Medical Informatics, University Medical Center Hamburg-Eppendorf, \\ Hamburg, Germany \\ a.schmidt-richberg@uke. uni-hamburg.de
}

\begin{abstract}
The computation of accurate motion fields is a crucial aspect in $4 \mathrm{D}$ medical imaging. It is usually done using a non-linear registration without further modeling of physiological motion properties. However, a globally homogeneous smoothing (regularization) of the motion field during the registration process can contradict the characteristics of motion dynamics. This is particularly the case when two organs slip along each other which leads to discontinuities in the motion field. In this paper, we present a diffusion-based model for incorporating physiological knowledge in image registration. By decoupling normal- and tangentialdirected smoothing, we are able to estimate slipping motion at the organ borders while ensuring smooth motion fields in the inside and preventing gaps to arise in the field. We evaluate our model focusing on the estimation of respiratory lung motion. By accounting for the discontinuous motion of visceral and parietal pleurae, we are able to show a significant increase of registration accuracy with respect to the target registration error (TRE).
\end{abstract}

\section{Introduction}

The accessibility of accurate motion fields is a precondition for many applications in medical imaging, e.g. for lung motion quantification in radiation therapy [1], wall movement analysis of the heart [2] or automatic contour propagation [3]. Motion estimation is usually done on the basis of $4 \mathrm{D}$ images by computing the displacements between time frames of the data sets using non-linear registration approaches.

While smoothing mechanisms are required during the registration process, they can contradict the physiology of the organ motion. This is especially the case when two objects slip along each other, which can be observed for example in the case of lung- 4 ] or liver motion [5].

Arising discontinuities at object boundaries are addressed by several approaches: In 4 finite element methods are used in order to simulate the physiology of respiration dynamics, point- and surface-based registration approaches are used in [6. While these allow to explicitly or implicitly incorporate boundary conditions, inner-organ information like bronchial or vessel trees are dismissed 
which results in an inaccurate registration of those structures. In [57] the problem of slipping organs is dressed implicitly by masking the background in order to prevent it from affecting the force calculation. However, this limits motion estimation to the object and provides no information about background motion.

In this paper, we present a novel smoothing mechanism that enables us to explicitly handle slipping motion at object boundaries. While other approaches use segmentations in order to model certain properties of objects (e.g. rigidity [8] or varying elasticities [9]) none addresses this specific physiological behaviour. Our model is closely related to the diffusive regularisation approach [10]. By decoupling normal- and tangential-directed smoothing we are able to estimate discontinuities at the object boundaries while maintaining smooth inner-object motion and preventing gaps at the borders.

The presented approach is not limited to a specific application. However, we apply it in the context of radiotherapy for the treatment of thoracic tumors, where the computation of accurate motion fields of the lung is a crucial part. In-depth knowledge about lung motion is needed to explicitly account for the respiration-driven movement of tumor and organs at risk during the treatment, for example in order to determine appropriate safety margins or dose accumulations 1. From the perspective of physiology, motion estimation is especially challenging because visceral and parietal pleurae are slipping along each other during breathing, thus creating discontinuities in lung and chest wall motion. Transferred to the registration problem, this behavior conflicts with common regularisation models which favor smooth motion fields. As a result, registration errors arise in particular near the lung borders [7].

This paper is organized as follows. We start introducing our model in section 2. In section 3] a detailed evaluation and discussion is given for the estimation of lung motion fields. We summarize our approach in section 4

\section{Methods}

When applying non-linear registration methods for the estimation of organ motion, usually no a-priori knowledge about the physiological process is taken into account. Treating all regions in the image as one homogeneous object often results in questionable motion estimates.

In this paper, we present a model for a normal-directed regularization. We incorporate knowledge about the physiology of organ motion by allowing object and background to slide along each other (see Fig. 1). In the process we assume a given segmentation of the object in one frame.

We proceed by presenting an automatic preprocessing step to refine the segmentations, what turns out to be useful in order to improve accuracy and stability of subsequent steps (Sect. 2.1). In Sect. 2.2 we briefly summarize the basics of the diffusive registration. We then introduce our model for the novel regularisation approach in Sect. 2.3. 


\subsection{Preprocessing of Segmentations}

In order to determine object boundaries we use segmentations of the object, i.e. of the lung in our particular case. These segmentations were generated by a semi-automatic procedure, consisting of a volume growing followed by a manual slice-by-slice correction of the boundaries by a clinical expert. Due to this generation process and reconstruction artifacts in the CT data (comp. 11]), the segmentations often show inaccuracies and a non-smoothness, especially in z-direction.

These shortcomings are particularly impedimental to the discrete calculation of the surface normals (comp. Sect. 2.3). Therefore, we apply a level set based refinement of the segmentations.

Let $\Gamma$ be the segmented object in the reference image and $\widetilde{\phi}: \Omega \mapsto \mathbb{R}$ the corresponding level set function, with $\Omega \subset \mathbb{R}^{3}$ denoting the image domain. We calculate an improved segmentation $\phi(x), \boldsymbol{x} \in \Omega$ by minimizing the following energy functional:

$$
\mathcal{J}_{\text {Seg }}[\phi]:=\mathcal{I}[\phi]+\mathcal{E}[R ; \phi]+\mathcal{A}[\widetilde{\phi} ; \phi]
$$

The internal energy $\mathcal{I}$ provides a smoothing of the boundary as defined in [12]. We chose a region based external energy $\mathcal{E}$ as proposed in 3 .

The third energy term

$$
\mathcal{A}[\widetilde{\phi} ; \phi]:=\frac{1}{2} \int_{\Omega} H_{\alpha}(\phi(\boldsymbol{x}))\|\widetilde{\phi}(\boldsymbol{x})-\phi(\boldsymbol{x})\|^{2} d \boldsymbol{x},
$$

where $H_{\alpha}$ denotes a differentiable approximation of the Heaviside function, prevents the segmentation from moving too far away from the manual segmentation. As a result, we obtain a segmentation with increased smoothness and accuracy.

\subsection{Diffusive Registration}

Let $R, T: \Omega \mapsto \mathbb{R}$ be two 3D Images (i.e. timeframes) of a $4 \mathrm{D}$ data set, called reference image $R(\boldsymbol{x})$ and target image $T(\boldsymbol{x})$ with the image domain $\Omega \subset \mathbb{R}^{3}$. The goal is to find a motion field $\boldsymbol{u}: \Omega \mapsto \mathbb{R}^{3}$ that minimizes the energy functional

$$
\mathcal{J}_{\text {Reg }}[\boldsymbol{u}]:=\mathcal{D}[R, T ; \boldsymbol{u}]+\mathcal{S}[\boldsymbol{u}]
$$

The distance measure $\mathcal{D}$ is used to measure the similarity between the reference image and the warped target image $T(\boldsymbol{x}-\boldsymbol{u}(\boldsymbol{x}))$ whereas the smoothing term $\mathcal{S}$ provides smooth motion fields.

A common choice for $\mathcal{S}$ is the diffusive registration [10

$$
\mathcal{S}^{\text {Diff }}[\boldsymbol{u}]:=\frac{1}{2} \sum_{l=1}^{3} \int_{\Omega}\left\|\nabla u_{l}(\boldsymbol{x})\right\|^{2} d \boldsymbol{x},
$$

where $u_{l}$ is the $l$-th component of $\boldsymbol{u}$. A smoothing is archieved by penalizing large gradients in the vector field. Providing results similar to those of the elastic regularisation, this term is much more efficient with respect to computation time 7 . 
For the minimization of the energy functional (1) an iterative approach is employed. According to the calculus of variations, a solver of (1) must fulfill the condition $\partial \mathcal{J} / \partial \boldsymbol{u}=0$. From the associated Euler-Lagrangian equation the following iterative update scheme is derived:

$$
\boldsymbol{u}^{(k+1)}=\boldsymbol{u}^{(k)}+\tau \boldsymbol{c}_{\mathcal{S}}\left(\boldsymbol{u}^{(k)}\right)+\tau \boldsymbol{f}_{\mathcal{D}}\left(\boldsymbol{u}^{(k)}\right) .
$$

The force term $\boldsymbol{f}_{\mathcal{D}}(\boldsymbol{u})$ corresponds to the specific distance measure $\mathcal{D}$. In this paper, we use

$$
\boldsymbol{f}_{\mathcal{D}}(\boldsymbol{u}):=\frac{R(\boldsymbol{x})-T(\boldsymbol{x}-\boldsymbol{u}(\boldsymbol{x}))}{\|\nabla R(\boldsymbol{x})\|^{2}+\kappa^{2}} \nabla R(\boldsymbol{x}),
$$

where $\kappa$ denotes a normalizer which is used in order to account for image contrast. This force term is closely related to Thirion's demons, the corresponding distance measure can be found in [10.

The correction term $\boldsymbol{c}_{\mathcal{S}}(\boldsymbol{u})$ is used to smooth the field according to the regularizer $\mathcal{S}$. For (2) we find $\partial \mathcal{S} / \partial \boldsymbol{u}=\Delta \boldsymbol{u}$ what leads to $\boldsymbol{c}_{\mathcal{S}}(\boldsymbol{u})=\Delta \boldsymbol{u}$.

\subsection{Model for a Directional-Dependent Regularisation}

In this work, we extend the diffusive regularisation by restricting inter-object smoothing to the normal direction. Based on a given segmentation $\phi(\boldsymbol{x})$ of an anatomical object, the goal is to incorporate knowledge about the physiology of its motion by allowing object and background to slip along each other. From a technical point of view, we want to allow discontinuities between the movement of object and background in tangential direction, while maintaining smoothness in normal direction (see Fig. 1).

Let $\boldsymbol{n}(\boldsymbol{x})=\nabla \phi(\boldsymbol{x}) /\|\nabla \phi(\boldsymbol{x})\|$ be the normal of the segmentation at a point $\boldsymbol{x}$. We proceed by splitting the motion in two parts: the normal-directed part $\boldsymbol{u}^{\perp}(\boldsymbol{x})=\langle\boldsymbol{u}(\boldsymbol{x}), \boldsymbol{n}(\boldsymbol{x})\rangle \boldsymbol{n}(\boldsymbol{x})$ and the tangential-directed part $\boldsymbol{u}^{\|}(\boldsymbol{x})=\boldsymbol{u}(\boldsymbol{x})-$ $\langle\boldsymbol{u}(\boldsymbol{x}), \boldsymbol{n}(\boldsymbol{x})\rangle \boldsymbol{n}(\boldsymbol{x})$. We can then rewrite eq. (2) as

$$
\mathcal{S}^{\text {Diff }}[\boldsymbol{u}]=\frac{1}{2} \sum_{l=1}^{3} \int_{\Omega}\left\|\nabla\left(u_{l}^{\perp}+u_{l}^{\|}\right)\right\|^{2} d \boldsymbol{x}=\frac{1}{2} \sum_{l=1}^{3} \int_{\Omega}\left\|\nabla u_{l}^{\perp}\right\|^{2}+\left\|\nabla u_{l}^{\|}\right\|^{2} d \boldsymbol{x},
$$
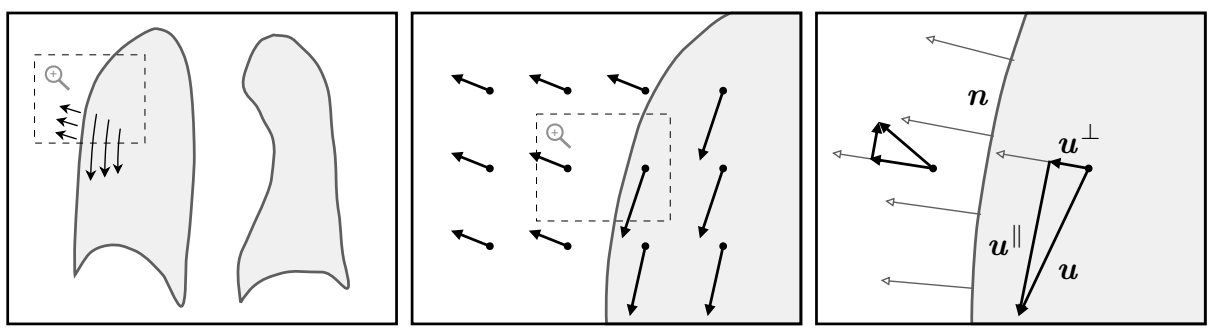

Fig. 1. In the center image the motion field along the border of the lung (left) is visualized. This is not smooth with respect to the diffusive regularization (2). By decoupling $\boldsymbol{u}^{\perp}$ and $\boldsymbol{u}^{\|}$we can demand the field to be smooth only in normal direction (right). 
whereat we assume $\boldsymbol{n}$ to be fixed, leading to $\sum_{l=1}^{3}\left\langle\nabla u_{l}^{\perp}, \nabla u_{l}^{\|}\right\rangle=0$. This assumption is feasible because the surfaces are smooth with respect to image spacing.

Based on (3), we define our new model in two steps. First, according to our assumptions, we want to achieve a comprehensive (i.e. inter-object) smoothing in normal direction while smoothing object and background separately in tangential direction. Thus, we split the domain of the energy terms: as before we define $\left\|\nabla u_{l}^{\perp}\right\|^{2}$ on the whole image domain $\Omega$, but we restrict $\left\|\nabla u_{l}^{\|}\right\|^{2}$ to the inside resp. outside of the object $\Gamma$, using Neumann boundary conditions. This leads to an interim formulation for a direction-dependent regularization (DDR)

$$
\mathcal{S}^{D D R^{\prime}}[\boldsymbol{u}]:=\frac{1}{2} \sum_{l=1}^{3}\left(\int_{\Omega}\left\|\nabla u_{l}^{\perp}\right\|^{2} d \boldsymbol{x}+\int_{\Gamma}\left\|\nabla u_{l}^{\|}\right\|^{2} d \boldsymbol{x}+\int_{\Omega / \Gamma}\left\|\nabla u_{l}^{\|}\right\|^{2} d \boldsymbol{x}\right) .
$$

In a second step, we include a weighting between the proposed regularization (4) and the common diffusive term (2) in order to restrict the calculation of (4) to the region close to the object borders. This is done because normals are only defined within this region. Moreover, it entails a computational benefit and allows us to admit a tangential smoothing of a certain amount. Following [9] we use the Dirac-shaped weighting function

$$
\delta(\phi(\boldsymbol{x}))=1-\frac{1}{1+\alpha c \exp ^{-\alpha \phi(\boldsymbol{x})^{2}}}
$$

in order to determine the object borders. The influence of the parameters $\alpha$ and $c$ on $\delta$ is depicted in [9]. This leads to the final energy term

$$
\begin{aligned}
\mathcal{S}^{D D R}[\boldsymbol{u}]:=\frac{1}{2} \sum_{l=1}^{3}( & \int_{\Omega} \delta(\phi)\left\|\nabla u_{l}^{\perp}\right\|^{2}+(1-\delta(\phi))\left\|\nabla u_{l}\right\|^{2} d \boldsymbol{x} \\
& \left.+\int_{\Gamma} \delta(\phi)\left\|\nabla u_{l}^{\|}\right\|^{2} d \boldsymbol{x}+\int_{\Omega / \Gamma} \delta(\phi)\left\|\nabla u_{l}^{\|}\right\|^{2} d \boldsymbol{x}\right) .
\end{aligned}
$$

As in Sect.2.2 we apply a variational framework in order to minimize the energy functional (11). Regarding the Euler-Lagrangian equation of (6) we find

$$
\boldsymbol{c}_{\mathcal{S}}(\boldsymbol{u})=\nabla \delta \nabla \boldsymbol{u}^{\perp}+\nabla(1-\delta) \nabla \boldsymbol{u}+\nabla_{\Gamma} \delta \nabla_{\Gamma} \boldsymbol{u}^{\|} .
$$

With $\nabla_{\Gamma}$ we denote the gradient being calculated only inside the object, using Neumann boundary conditions. In the background, $\nabla_{\Omega / \Gamma}$ is used respectively. N.B.: we assume the normals $\boldsymbol{n}$ to be independent of the position $\boldsymbol{x}$ for the calculation of this derivative. The inclusion of the weighting function $\delta$ in (6) leads to terms related to an anisotropic diffusion which are implemented efficiently according to 13 .

\section{Results}

The evaluation is based on 4D CT data sets of 12 lung cancer patients acquired during free breathing. Data sets are reconstructed using an optical flow based 

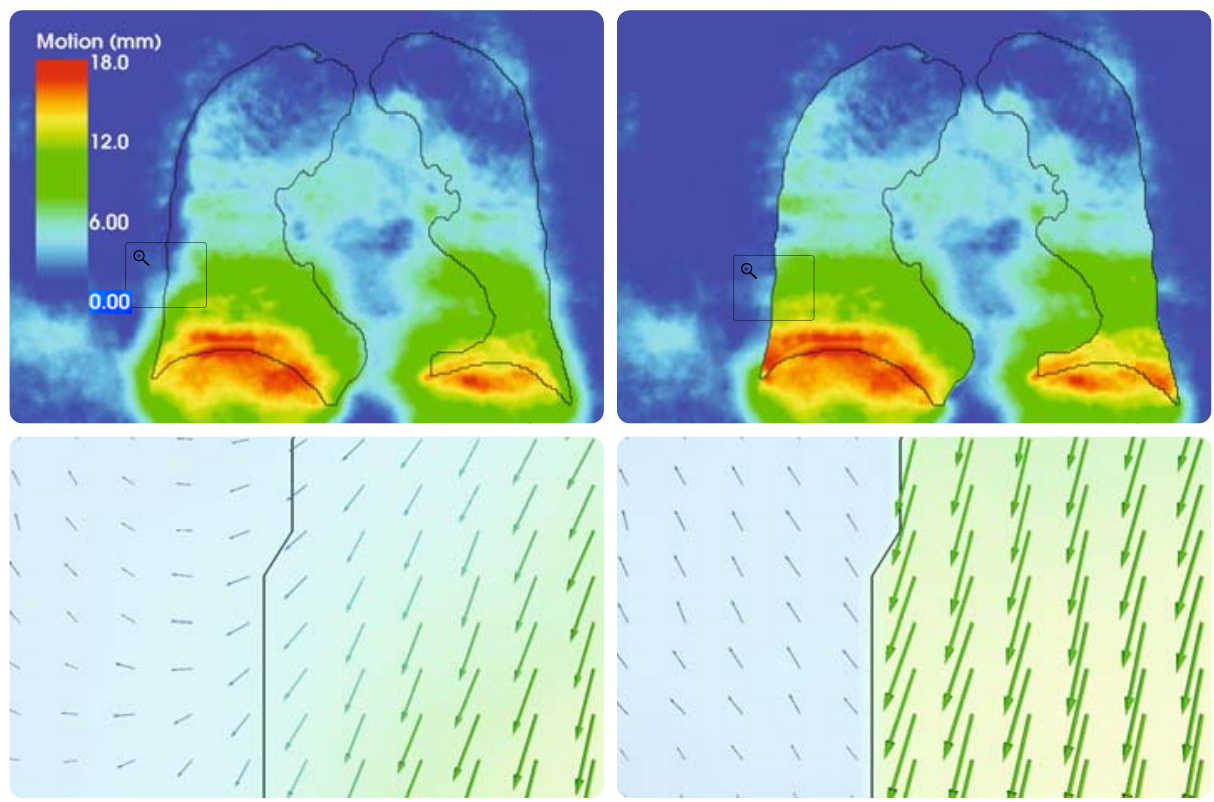

Fig. 2. Top: Motion magnitudes after a registration with diffusive (left, (2)) and directional-dependent regularisation (right, (6) ). Bottom: The estimated motion field close to the lung border for the diffusive (left) and the directional-dependent (right) approach. For further explanation please refer to the text.

method 11. Spatial resolution is $0.98 \times 0.98 \times 1.5 \mathrm{~mm}$. Each data set consists of 3D CT images at 10 to 14 different breathing phases of which we chose endinspiration (EI) as reference image $R$ and end-expiration (EE) as target image $T$. The segmentations are obtained as described in Sect. 2.1.

In Fig. 2, a qualitative comparison between the registration with the standard diffusive regularizer (2) and the direction-dependent regularizer (6) is given. In order to improve results as well as computational efficiency, a multi-resolution strategy was applied on both approaches. While the motion fields in the inside of the lung are almost identical, considerable differences occur near to the lung borders. In this area, the motion estimated by the directional-dependent approach satisfies the expectation derived from respiration physiology (comp. Fig. 1) and is not impaired by the marginal movement of the background as it is the case with the diffusive registration.

For a quantitative analysis, a medical expert identified inner lung landmarks (prominent bifurcations of the bronchial tree and the vessel tree) in the CT data at EI and EE. Landmarks are differentiated into landmarks located in the middle of the lung (20 landmarks each lung), near the lung borders (15 landmarks), and near the tumor (10 each lung tumor).

Registration accuracy is quantified as target registration error (TRE), i.e. the mean Euclidean distance between reference landmarks and target landmarks a) before registration, b) after a diffusive registration, c) after a masked 
Table 1. Target registration errors (TRE) and variations in $\mathrm{mm}$. The last row lists the statistical significance of the improvement of direction-dependent vs. diffusive regularisation, using levels of $p<0.05(+), p<0.01(++)$ and $p<0.001(+++)$.

\begin{tabular}{c|cccc|cc} 
Pat. & w/o Reg. & Diffusive & Masked & Direction-Dep. & $p$-value & Significance \\
\hline 01 & $4.25 \pm 6.5$ & $1.08 \pm 0.7$ & $1.06 \pm 0.6$ & $1.04 \pm 0.4$ & 0.422 & $=$ \\
02 & $6.26 \pm 22.7$ & $1.73 \pm 1.9$ & $1.21 \pm 0.4$ & $1.21 \pm 0.4$ & $<0.001$ & +++ \\
03 & $5.45 \pm 7.4$ & $1.73 \pm 3.0$ & $1.65 \pm 2.8$ & $1.48 \pm 1.3$ & 0.011 & + \\
04 & $6.20 \pm 4.6$ & $1.74 \pm 1.7$ & $1.39 \pm 0.7$ & $1.44 \pm 0.9$ & 0.003 & ++ \\
05 & $6.79 \pm 14.0$ & $1.80 \pm 2.3$ & $1.50 \pm 1.4$ & $1.55 \pm 1.4$ & 0.012 & + \\
06 & $6.44 \pm 9.5$ & $1.67 \pm 1.3$ & $1.36 \pm 0.6$ & $1.43 \pm 1.0$ & 0.011 & + \\
07 & $4.31 \pm 9.8$ & $1.41 \pm 1.3$ & $1.35 \pm 0.6$ & $1.35 \pm 0.7$ & 0.025 & + \\
08 & $10.76 \pm 60.8$ & $2.48 \pm 10.2$ & $1.43 \pm 1.6$ & $1.49 \pm 2.2$ & $<0.001$ & +++ \\
09 & $6.40 \pm 46.0$ & $2.80 \pm 11.5$ & $2.52 \pm 10.8$ & $2.40 \pm 7.2$ & 0.002 & ++ \\
10 & $6.06 \pm 25.4$ & $1.37 \pm 2.5$ & $1.10 \pm 0.4$ & $1.19 \pm 0.9$ & 0.063 & $=$ \\
11 & $7.98 \pm 28.0$ & $2.07 \pm 6.9$ & $1.69 \pm 2.8$ & $1.71 \pm 3.6$ & 0.007 & ++ \\
12 & $8.31 \pm 40.9$ & $2.14 \pm 5.4$ & $1.64 \pm 2.8$ & $1.52 \pm 1.6$ & $<0.001$ & +++ \\
\hline
\end{tabular}

diffusive registration and d) after the directional-dependent registration. The masked registration is done following for example [5/7] by defining $\boldsymbol{f}_{\mathcal{D}}(\boldsymbol{x}):=0$ for all $\boldsymbol{x} \in \Omega / \Gamma$. In order to quantify the improvement of the directional-dependent approach with relation to the standard diffusive registration, we further perform a two-sample $t$-test following [14]. The results are given in Table 1.

The presented directional-dependent model outperforms the diffusive approach with respect to the TRE for all examined patients. In 10 out of 12 cases, the results are statistically significant. As expected, the improvement is considerable especially for landmarks near the lung border. Neither the directional-dependent nor the masked registration proves to be superior with regard to registration accuracy. However, the directional-dependent approach allows a closed mathematical formulation and provides a motion estimation for the whole image domain while the masked registration limits calculation to the lung.

\section{Discussion and Conclusion}

We presented a new registration approach that enables us to incorporate physiological knowledge into the smoothing mechanism. In order to model the slipping motion of objects, regularization procedure was decoupled into normal and tangential direction. While smoothing object and background separately in normal direction, tangential-directed smoothing was performed comprehensively. In addition, we presented an approach for an automatic refinement of the segmentations which is substantial to warrant smooth normals.

The parameters $c$ and $\alpha$ in (5) can be determined generally and are not very sensitive to the specific application. In addition, no further parameters occur compared to the standard approach.

We validated the approach for motion field estimation of the lung on 12 clinical data sets. The presented directional-dependent regularization significantly 
outperformed the common diffusive approach with respect to the TRE and provided results similar to those of the masked registration. However, with the directional-dependent registration we introduced a model for the estimation of the whole image domain, which also includes neighboring structures.

\section{References}

1. Keall, P.J., Mageras, G.S., Balter, J.M., et al.: The management of respiratory motion in radiation oncology report of AAPM Task Group 76. Med. Phys. 33(10), 3874-3900 (2006)

2. Chandrashekara, R., Mohiaddin, R.H., Rueckert, D.: Cardiac motion tracking in tagged MR images using a 4D B-spline motion model and nonrigid image registration. In: Proc. ISBI, vol. 1, pp. 468-471 (2004)

3. Schmidt-Richberg, A., Ehrhardt, J., Handels, H.: Integrated Segmentation and Non-Linear Registration for Organ Segmentation and Motion Field Estimation in 4D CT Data. Methods Inf. Med. (in press, 2009)

4. Werner, R., Ehrhardt, J., Schmidt, R., Handels, H.: Patient-Specific Finite Element Modeling of Respiratory Lung Motion using 4D CT Image Data. Med. Phys. 35(5), 1500-1511 (2009)

5. von Siebenthal, M., Szekely, G., Gamper, U., Boesiger, P.: 4D MR imaging of respiratory organ motion and its variability. Phys. Med. Biol. 52(6), 1547-1564 (2007)

6. von Berg, J., Barschdorf, H., Blaffert, T., Kabus, S.: Surface based cardiac and respiratory motion extraction for pulmonary structures from multi-phase CT. In: Proc SPIE, vol. 6511, p. 65110Y (2007)

7. Werner, R., Ehrhardt, J., Schmidt-Richberg, A., Handels, H.: Validation and comparison of a biophysical modeling approach and non-linear registration for estimation of lung motion fields in thoracic 4D CT data. In: Proc SPIE (2009)

8. Pitiot, A., Guimond, A.: Geometrical regularization of displacement fields for histological image registration. Med. Image Anal. 1(12), 16-25 (2008)

9. Kabus, S.: Multiple-Material Variational Image Registration. PhD thesis, Institut für Mathematik, Universität zu Lübeck (2006)

10. Modersitzki, J.: Numerical Methods for Image Registration. Oxford University Press, Oxford (2004)

11. Ehrhardt, J., Werner, R., Säring, D., Lu, W., Low, D.A., Handels, H.: An optical flow based method for improved reconstruction of 4D CT data sets acquired during free breathing. Med. Phys. 34(2), 711-721 (2007)

12. Chan, T.F., Vese, L.A.: Active contours without edges. IEEE Trans. Image Process 10(2), 266-277 (2001)

13. Weickert, J., Romeny, B., Viergever, M.: Efficient and reliable schemes for nonlinear diffusion filtering. IEEE Trans Image Process 7(3), 398-410 (1998)

14. Sarrut, D., Delhay, S., Villard, P., et al.: A comparison framework for breathing motion estimation methods from 4-D imaging. IEEE Trans. Med. Imag. 26(12), 1636-1648 (2007) 